\title{
Erratum to: Marker utility of transposable elements for plant genetics, breeding, and ecology: a review
}

\author{
Neha Samir Roy $^{1} \cdot$ Ji-Yeong Choi $^{1} \cdot$ Sung-Il Lee $^{1} \cdot$ Nam-Soo Kim $^{1,2}$
}

Published online: 17 March 2015

(C) The Genetics Society of Korea and Springer-Science and Media 2015

\section{Erratum to: Genes Genom (2015) 37:141-151 DOI 10.1007/s13258-014-0252-3}

Regrettably, the original version of the article contains a few errors. These errors should be corrected as follows:

1. In page 143 of the published article, the line 16-18 should be corrected as follow: the gene order of $T y l$ copia retrotransposons is PR-INT-RT-RH, whereas that of Ty3-gypsy retrotransposons is PR-RT-RH-INT.

2. Figure 1 should be corrected as follow.

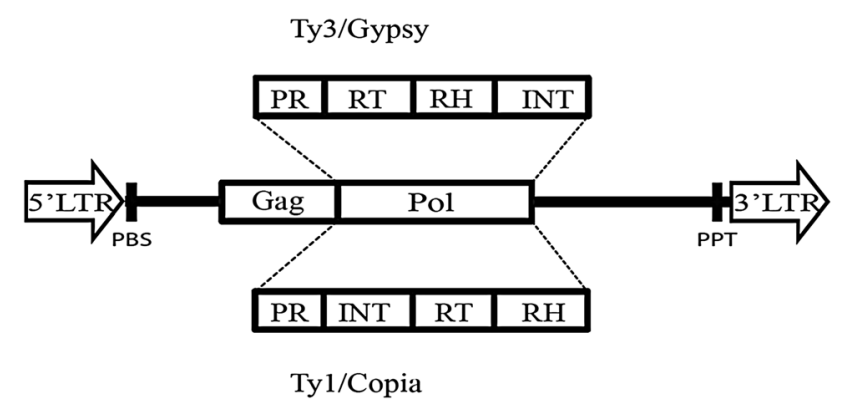

Fig. 1 Structure of the Ty1-copia and Ty3-gypsy retrotransposons. Both LTR retrotransposons have long terminal repeats at both ends. The gene order at the Pol region is different between the Tyl-copia and $T y 3$-gypsy elements. $P B S$ primer binding site, $P P T$ poly purine track, $P R$ protease, $R H$ RNase $\mathrm{H}, I N T$ integrase, $R T$ reverse transcriptase
The online version of the original article can be found under doi: 10.1007/s13258-014-0252-3.

Nam-Soo Kim

kimnamsu@kangwon.ac.kr

1 Department of Molecular Biosciences, Kangwon National University, Chucheon 200-701, Korea

2 Institute of Bioscience and Biotechnology, Kangwon National University, Chucheon 200-701, Korea 